\title{
Impact of line and control parameters on Droop Stability in Inverters for Distributed Generation
}

\author{
A. Bolzoni Student Member, IEEE, G. M. Foglia, L. Frosio, M. F. Iacchetti, Member, IEEE, \\ R. Perini Member, IEEE
}

\begin{abstract}
The paper analyzes stability conditions and design requirements for the control parameters in droop-controlled inverters. The analysis focusses on a single inverter unit with AC (output) filters and interface transformer, as this may be regarded as the elementary component of a distributed generation system. Through a process of thought simplifications and manipulations, the linearized full state-space model is transformed into a unique single-input, single-output system comprising several nested loops. The resulting analytical model in terms of transfer functions has enough "structure" to represent interactions between the different actors of the overall control, such as droop linear and derivative terms, virtual impedance and voltage controllers. Stability conditions for the corresponding parameters are then derived analytically. Experimental tests are carried out on the complete system to check the reliability of the proposed model.
\end{abstract}

Index Terms-Microgrids, droop control, virtual impedance, system stability, modal analysis.

\section{NOMENCLATURE}

$m, n\left(m_{d}, n_{d}\right), m_{\text {rest }}, n_{\text {rest }}$ : linear (derivative) droop coefficients of the inverter under study, of the rest of the network [p.u.]; $H_{i}$ : grid-current compensation gain in the voltage loop; $k_{p V}, k_{i V}, T_{i V}=k_{p V} / k_{i V}$ : parameters of the voltage PI controller; $p=d / d t$ : time derivative operator;

$p_{o},\left(P_{o}\right), q_{o},\left(Q_{o}\right)$ : instant. (average) real, reactive power;

$R_{f}, L_{f}, C_{f}$ : filter resistance / inductance / capacitance [p.u.];

$R_{t}, L_{t}\left(R_{v}, L_{v}\right)$ : line (virtual) resistance, inductance [p.u.];

$T_{t}=L_{t} /\left(R_{t} \omega_{b}\right)$ : time constant of the line impedance [s];

$T_{p}$ : time constant of the filter for the power $p_{o}, q_{o}$;

$\tau_{d m}=m_{d} /\left(m+m_{\text {rest }}\right) ; \tau_{d n}=n_{d} /\left(n+n_{\text {rest }}\right)$;

$\omega$ : inverter angular frequency [p.u.];

$\omega_{b}, \omega_{p c c}, \omega_{n}, \omega_{s}$ : base, point of common connection (pcc), rated and generic angular frequency;

$\omega^{*}, V^{*}, \omega_{\text {rest }}{ }^{*}, V_{\text {rest }}{ }^{*}$ : no-load angular frequency and voltage in the droop control of the inverter and of the rest of the grid;

$\omega_{c I},\left(\omega_{c I n}\right)$ : cut-off frequency (rated value) of the current loop.

\section{INTRODUCTION}

IN dispersed generation systems when there is no communication between the sources [1], stable operation of paralleled inverters and scheduled power sharing may be

A. Bolzoni, G.M. Foglia, R. Perini are with Dip.to di Energia, Politecnico di Milano, via La Masa 34, 20156 Milano, Italy.

L. Frosio is with EPS, via Grazzini 14, 20158 Milano, Italy.

M.F. Iacchetti is with School of Electrical and Electronic Engineering, University of Manchester (UK).

[alberto.bolzoni, gianmaria.foglia, roberto.perini]@polimi.it. achieved through droop control [2]. Either linear or piecewise droop characteristics are usually implemented [3], but nonlinear curves have also been proposed [4]. As for the linear case, two additional terms proportional to power derivatives are often introduced in order to improve stability [5], [6].

Low voltage microgrids are characterized by line reactance to resistance ratio less than one [7] or around one if the decoupling transformer is taken into account. This implies that active and reactive powers are not inherently decoupled [8]. Several methods have been proposed to solve this problem. In [9] a reference frame rotation is proposed, so that transformed active and reactive powers in the new reference frame only depend on load angle and voltage respectively. In [10] a technique based on inverted droop equations is proposed for highly resistive networks. In [11] the virtual impedance is introduced for a single phase network. For three-phase systems, a steady-state formulation of the virtual impedance is usually considered [12]. This is because the power decoupling is considered not important during transients. As discussed in [13], the concept can be extended to non-linear loads by introducing several steady-state virtual impedances in multiple reference frames, each for a given harmonic component. Transient virtual impedance formulations, namely involving time derivatives of currents, have also been proposed [14].

The virtual impedance changes the magnitude and the phase of the reference voltages due to the droop control. In [15] a virtual angle applied to the reference voltage is introduced, so as not to change the magnitude of the reference voltage.

As for the design of the virtual impedance, [12] proposes a method based on some decoupling coefficients: they report the sensitivity of the real power to the load angle and to the voltage referred to the sensitivity of the reactive power to the same quantities. The design approach proposed in [11] derives the virtual impedance value starting from the inverter output impedance, which is a function of the LC filter, voltage and current control parameters.

Small-signal analysis is a well-established tool for studying stability of different configurations of microgrids [16]-[19]. In all these schemes, however, stability is generally assessed by numerical eigenvalue computation using detailed state-space models, which do not give direct analytical insight into the stability conditions for the control parameters.

To the best authors' knowledge, no comprehensive models on transfer functions have been proposed to analytically show the impact of the control parameters on stability and to tune the virtual impedance gains. Simplified models described in literature (e.g. [6]) are usually of low order, and inevitably neglect multiple interactions between different control blocks.

Such a lack is addressed in this paper, where closed-form 
transfer functions are derived with the aim to analytically show the effect of the main control parameters on stability. Conversely to extremely simplified approaches which enucleate the droop from the system, the proposed reduced model has enough "structure" to represent interactions between different elements across the overall control, such as droop linear and derivative terms, virtual impedance and voltage controllers. Stability conditions for the corresponding parameters are then derived analytically. The analysis considers an inverter in parallel with other units and working in a quasi-no-load operating condition, as this is the critical condition for stability. The paper aims at giving some guidelines for the design of control parameters and for the stability analysis. Though the on-line estimation of the grid impedance is beyond the scope of this paper, the suggested design criteria may also be applied in the adaptive tuning of the virtual impedance to ensure stability under large gridimpedance variations.

The paper is divided as follows. In Sec. III a mathematical description of the basic model is given, taking into account the voltage and current controllers and the steady-state virtual impedance. In Sec. IV, a representative model in terms of transfer functions is derived together with closed-loop block diagrams. These are used in Sec. V, to study the stability from an analytical viewpoint, discussing the impact of control parameters and deriving stability conditions and design criteria. Sec VI compares the analytically-predicted dominant poles with some experimental tests.

\section{SYSTEM DESCRIPTION AND FUll ORDER MODEL}

\section{A. Hypotheses and limitations.}

The system under study is a microgrid supplied by several droop-controlled inverters (Fig. 1). The analysis is performed just on one inverter unit, comprising the output filter and connection transformer. A damping resistor may be connected in series to the filter capacitor, to avoid parallel resonance. The inductor $L_{t}-R_{t}$ represents the interface transformer and the line. All the other inverters are represented as a simple voltage source whose amplitude $V_{p c c}$ and angular frequency $\omega_{p c c}$ change according to the droop control.

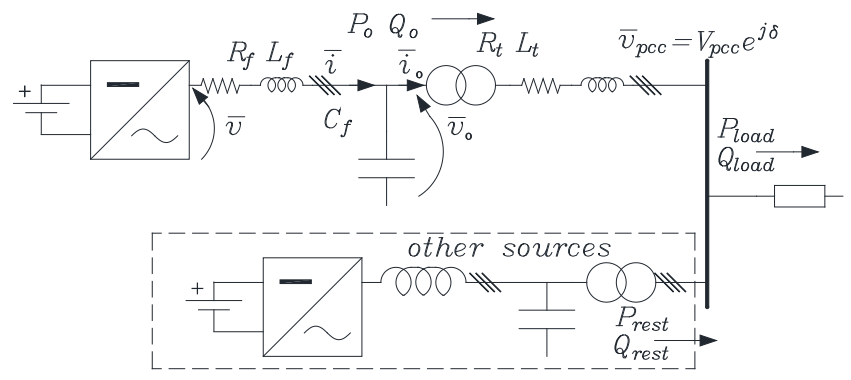

Fig. 1. Scheme of the analyzed system. An inverter is connected in parallel to other inverters in a microgrid. All inverters are droop controlled.

The equations of the whole system are expressed in per unit (p.u.) and referred to a dq frame, where the $d$-axis is aligned to the reference voltage $\bar{v}_{o}^{r e f}$ and $p=d / d t$ is the time derivative operator. Only time $t$ is not in p.u.: this is why the derivative operator $p$ is divided by the reference angular frequency $\omega_{b}$. Equations $(1) \div(8)$ deal with the inverter under study. Vector equations (1), (2) and (3) refer to the LCL filter. Equations (4) and (5) refer to the droop control system, where the angular frequency $\omega$ and the reference voltage $\bar{v}_{o}^{r e f}$ depend on the average output real $P_{o}$ and imaginary $Q_{o}$ powers and their derivatives, respectively, through the droop coefficients $m, m_{d}$, $n, n_{d}$. Both $P_{o}$ and $Q_{o}$ are averaged with a first order filter with time constant $T_{p}$ (cut off frequency $T_{p}{ }^{-1}$ ). $V^{*}$ and $\omega^{*}$ are the noload voltage and angular frequency. Equations (6) and (7) report the current and voltage PI control models, respectively. The coefficient $H_{i}$ is positive and less than unity: its purpose is to compensate for the output current in the real system. In particular, (7) includes the term due to the virtual impedance $\left(R_{v}, L_{v}\right)$ in steady state. Equation (8) represents the dynamic of the load angle $\delta$, defined as the angle between the PCC voltage $\bar{v}_{p c c}=V_{p c c} e^{j \delta}$ and the voltage vector $\bar{v}_{o}^{r e f}$ (see (3)): $\omega_{p c c}$ and $\omega$ are the point of common connection (PCC) and inverter angular frequency in p.u., respectively. Equations (9) represent the droop laws for the rest of the sources, and (10) come from the power balance.

$$
\begin{gathered}
\bar{v}-\bar{v}_{o}=R_{f} \bar{i}+\left(L_{f} / \omega_{b}\right) p \bar{i}+j \omega L_{f} \bar{i} \\
\left(C_{f} / \omega_{b}\right) p \bar{v}_{o}+j \omega C_{f} \bar{v}_{o} \cong\left(\bar{i}-\bar{i}_{o}\right) \\
\bar{v}_{o}=V_{p c c} e^{j \delta}+R_{t} \bar{i}_{o}+\left(L_{t} / \omega_{b}\right) p \bar{i}_{o}+j \omega L_{t} \bar{i}_{o} \\
\omega=\omega^{*}-\left(m+m_{d} p\right) P_{o}=\omega^{*}-\frac{\left(m+m_{d} p\right)\left(v_{o d} i_{o d}+v_{o q} i_{o q}\right)}{1+T_{p} p} \\
v_{o d}^{r e f}=V^{*}-\left(n+n_{d} p\right) Q_{o}=V^{*}-\frac{\left(n+n_{d} p\right)\left(v_{o q} i_{o d}-v_{o d} i_{o q}\right)}{1+T_{p} p} \\
\bar{v}^{\text {ref }}=\left(k_{p I}+k_{i I} / p\right)\left(\bar{i}^{r e f}-\bar{i}\right)+\bar{v}_{o}+j \omega L_{f} \bar{i} \\
=\left(H_{i} \bar{i}_{o}\right)+\left(k_{p V}+\frac{k_{i V}}{p}\right)\left(\bar{v}_{o}^{\text {ref }}-\bar{v}_{o}-\left(R_{v}+j \omega L_{v}\right) \bar{i}_{o}\right) \\
p \delta=\omega_{b}\left(\omega_{p c c}-\omega\right) \\
\omega_{p c c}=\omega_{\text {rest }}^{*}-m_{\text {rest }} P_{\text {rest }} \quad V_{p c c}=V_{\text {rest }}^{*}-n_{\text {rest }} Q_{\text {rest }} \\
P_{\text {rest }}+P_{o} \cong P_{\text {load }}
\end{gathered}
$$

A scheme of the adopted control system is shown in Fig. 2 .

\section{B. Main data.}

Reference will be made to an inverter source whose data are reported in p.u. in Table I. The base quantities are the apparent power $A_{b}=A_{n}$, the voltage $V_{b}=V_{n}$ and the angular frequency $\omega_{b}=\omega_{n}$. 
TABLE I

MAIN DATA OF THE INVERTER SOURCE UNDER STUDY.

\begin{tabular}{c|c}
\hline Rated $V_{n}[\mathrm{~V}], I_{n}[\mathrm{~A}], A_{n}[\mathrm{kVA}], \omega_{n}[\mathrm{rad} / \mathrm{s}]$ & $200 ; 7 ; 2.4 ; 2 \pi 50$ \\
\hline $\begin{array}{c}\text { Inverter max real } P_{\max } \text { and reactive } Q_{\max } \\
\text { power }\end{array}$ & $A_{n} ; 0.6 A_{n}$ \\
\hline$R f, L f, C f$ [p.u.] & $0.0073 ; 0.045 ; 0.052$ \\
\hline$R_{t} ; L_{t}$ [p.u.] & $0.049 ; 0.024$ \\
\hline $\begin{array}{c}\text { Current loop } \omega_{c l}, \text { voltage loop } \omega_{c V} \text { cross - } \\
\text { over frequency [rad/s] }\end{array}$ & $2 \pi 350 ; 2 \pi 35$ \\
\hline$H_{i}, k_{p V}, k_{i V}$ [p.u.], [p.u.], [p.u./s] & $0.90 ; 1.41 ; 733$ \\
\hline Time constant $T_{p}$ droop power filter [s] & 0.10 \\
\hline
\end{tabular}

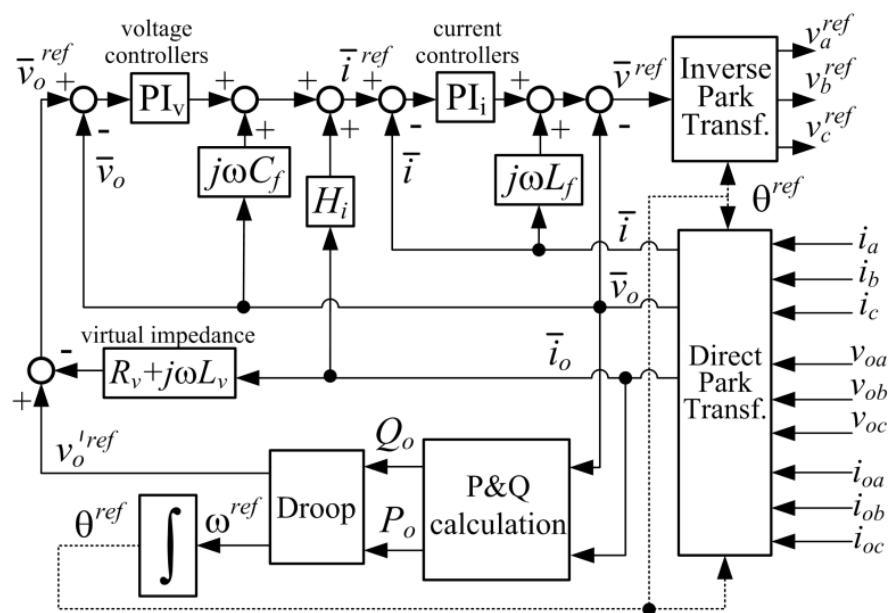

Fig. 2. Structure of the proposed cascaded control system: an internal current controller and an external voltage one; then the droop control.

The parameters in Table I may be considered of general validity. In fact, a reasonable criterion has been used to design the LC filter, namely: $4.5 \%$ series voltage drop, $5 \%$ capacitive reactive power due to the capacitors [20].

Also the assumed ratio $L_{t} / R_{t} \approx 0.5$ is reasonable for a low voltage line including a decoupling transformer. The virtual impedance is chosen as a guess value so as to decouple real and reactive powers, increasing the ratio $L / R$. According to [12], by introducing virtual impedance $L_{v} \approx L_{t}, R_{v}=-0.75 R_{t}$, the reported decoupling coefficients $\left(\partial P_{o} / \partial \delta\right) /\left(\partial Q_{o} / \partial \delta\right)$ and $\left(\partial Q_{o} / \partial v_{o d}{ }^{r e f}\right) /\left(\partial P_{o} / \partial v_{o d}{ }^{r e f}\right)$ pass from 0.5 to 4 . Pros and cons of a negative $R_{v}$ will be illustrated in Sec. V .

The compensating term $H_{i} \bar{i}_{0}$ can only reduce the excursion of the voltage controller, but of course it does not affect the inverter ratings. Usual values of $H_{i}$ are within the range $0.70 \div 0.90$ [5], [21].

The cross-over frequency $\omega_{c I}$ of the current loop has been assumed equal to $\omega_{c I}=2 \pi \cdot 350 \mathrm{rad} / \mathrm{s}$. The design of the voltage controllers in the $d$ - and $q$-axis is carried out by Bode diagrams, considering a SISO (Single Input, Single Output) system, i.e. without mutual coupling. Let the cross-over frequency $\omega_{c V}$ of the external voltage loop range from $0.05 \omega_{c I}$ up to $0.15 \omega_{c}$; the design of the PI voltage controller implies that the range of the integral term $k_{i V}$ is $600 \div 1160$ p.u./s, while the range of the integral time $T_{i V}=\left(k_{p V} / k_{i V}\right)$ is $5.0 \div 2.0$ ms. These values will be used in the following considerations, together with rated values reported in Table I.

The time constant $T_{p}$ of the droop power system is usually quite high, equal to some periods of the line frequency.

\section{SIMPLIFIED MODEL}

\section{A. Simplified model equations.}

The current loop (equations (1), (6)) has a large cut-off frequency. Thus, it is represented by a first order time delay, $\omega_{c I}{ }^{-1}$, in order to study only the dominant poles of the system, simplify the analysis and derive manageable expressions:

$$
\bar{i}=\bar{i}_{r e f}\left[\omega_{c I} /\left(s+\omega_{c I}\right)\right] .
$$

Introducing (7) into (11) and then into (2) yields (12), where some approximations can be invoked to simplify the coefficients, limiting the frequency analysis within a few multiples of the voltage cut-off frequency $\omega_{c V}$, so as to include the dominant poles:

$$
\bar{v}_{o}=G_{1}(p) \bar{v}_{o}^{r e f}-H_{2}(p) \bar{i}_{o}-j H_{2 b}(p) \omega \bar{v}_{o}-j H_{2 c}(p) \omega \bar{i}_{o}
$$

Transfer functions in (12) are

$$
\begin{gathered}
G_{1}(p) \approx 1, \quad H_{2 b}(p) \approx 0, \quad H_{2 c}(p) \approx L_{v} \\
H_{2}(p) \approx \frac{R_{v}\left[1+\left(T_{i V}+\frac{\left(1-H_{i}\right)}{k_{i V} R_{v}}\right) p+\frac{p^{2}}{R_{v} k_{i V} \omega_{c I}}\right]}{\left(1+T_{i V} p\right)}
\end{gathered}
$$

A linearization of (12), (3)-(5) and (8)-(10) around a stable condition is performed: the stable condition is expressed by capital letters or superscript $\left({ }^{0}\right)$, and the small variations are analyzed. In steady state conditions, the vector $\bar{v}_{O}$ lies on the $\mathrm{d}$-axis and power flows from the inverter to the PCC: thus $V_{\text {od }} \approx 1$ p.u.; $V_{\text {oq }}=0$; $\delta^{0}<0$. Then:

$$
\begin{aligned}
& \Delta \bar{v}_{o}=\Delta \bar{v}_{o}^{r e f}-H_{2}(p) \Delta \bar{i}_{o}-j L_{v}\left(\omega^{0} \Delta \bar{i}_{o}+\Delta \omega \bar{I}_{o}\right) \\
& \Delta \bar{v}_{O}-\Delta V_{p c c} e^{j \delta^{0}}-j V_{p c c}^{0} e^{j \delta^{0}} \Delta \delta= \\
&=R_{t}\left(1+p T_{t}\right) \Delta \bar{i}_{o}+j L_{t}\left(\omega^{0} \Delta \bar{i}_{o}+\bar{I}_{o} \Delta \omega\right)
\end{aligned}
$$

where: $\quad T_{t}=L_{t} /\left(\omega_{b} R_{t}\right), G_{2}(p)=\left[R_{t}\left(1+p T_{t}\right)\right]^{-1}$

$$
\begin{gathered}
\Delta \omega=\Delta \omega^{*}-\left(\Delta p_{o}\right)\left[\left(m+m_{d} p\right) /\left(1+T_{p} p\right)\right] \\
\Delta p_{o}=\left(\Delta v_{o d} I_{o d}+V_{o d} \Delta i_{o d}+\Delta v_{o q} I_{o q}\right) \\
\Delta v_{o d}^{r e f}=\Delta V^{*}-\left(\Delta q_{o}\right)\left[\left(n+n_{d} p\right) /\left(1+T_{p} p\right)\right] \\
\Delta q_{o}=\left(\Delta v_{o q} I_{o d}-\Delta v_{o d} I_{o q}-V_{o d} \Delta i_{o q}\right) \\
p(\Delta \delta)=\omega_{b}\left(\Delta \omega_{p c c}-\Delta \omega\right) \\
\Delta \omega_{p c c}=-m_{\text {rest }} \Delta P_{\text {rest }} \quad \Delta V_{p c c}=-n_{\text {rest }} \Delta Q_{\text {rest }}
\end{gathered}
$$




$$
\Delta P_{\text {rest }}+\Delta P_{o} \cong \Delta P_{\text {load }} \quad \Delta Q_{\text {rest }}+\Delta Q_{o} \cong \Delta Q_{\text {load }}
$$

These equations can be represented by the diagram in Fig. 3, which includes some feedback loops. In order to help the understanding, the block diagram highlights the fictitious input $\Delta v_{\mathrm{oq}}{ }^{\text {ref }}=0$ forcing the orientation. The approach proposed in this paper allows the whole system to be transformed into a series of simpler subsystems of a Single-Input Single-Output (SISO) type with no interlaced connections, so that a stability analysis can be analytically carried out providing design criteria.

\section{B. Closed loops for the stability analysis.}

From now on, the complex frequency $s$ will replace the derivative operator $p$ and the generic angular frequency will be $\omega_{s}$.

The analysis is bounded to the dominant poles: thus only the low frequency range is studied. Some simplifications can be adopted in a quasi-no-load condition by considering $V_{\text {od }} \approx 1$ p.u., $V_{\text {oq }} \approx 0, I_{\text {oq }} \approx 0, \delta^{0}<0$ but small, $L_{\nu} \approx L_{t}$, yielding the diagram in Fig. 4.

An approximation of the transfer function $F_{2}$ representing the closed loop $G_{2}$ and $H_{2}$ in Fig. 3 is:

$$
F_{2}(s)=\frac{G_{2}(s)}{1+G_{2}(s) H_{2}(s)} \approx \frac{\left(R_{v}+R_{t}\right)^{-1}\left(1+s T_{i V}\right)}{\left(1+s T_{2 a}\right)\left(1+s T_{2 b}\right)}
$$

where $T_{2 a}>T_{2 b}$;

$$
\begin{aligned}
& T_{2 a}^{-1} \approx \frac{\left(R_{v}+R_{t}\right)}{T_{i V}\left(R_{V}+R_{t}\right)+L_{t} / \omega_{b}+\left(1-H_{i}\right) / k_{i V}} \\
& T_{2 b}^{-1} \approx \frac{\left(R_{v}+R_{t}\right) T_{i V}+L_{t} / \omega_{b}+\left(1-H_{i}\right) / k_{i V}}{\left(k_{i V} \omega_{c I}\right)^{-1}+T_{i V} L_{t}}
\end{aligned}
$$

( $T_{i V}, K_{i V}, \omega_{c I}$ ), on the virtual resistance $R_{v}$, but not on the virtual inductance $L_{v}$.

Finally, some independent SISO closed loops have been derived. They link inputs $\Delta v_{\mathrm{oq}}{ }^{\text {ref }}, \Delta V^{*}$ and $\Delta \omega^{*}$ to $\Delta v_{\text {od }}{ }^{\text {ref }}$ and $\Delta \omega$. This block diagram (Fig. 4) is the starting point to analytically find the stability conditions of the system. It will be used hereafter to analyze the influence of line and control parameters on the stability.

\section{STABILITY ANALYSIS AND DESIGN CRITERIA.}

\section{A. Stability analysis}

Fig. 3 contains a first closed loop including $G_{2}(s)$ and $H_{2}(s)$. The open loop transfer function at low frequencies is:

$$
L_{2}(s)=G_{2}(s) H_{2}(s)=\frac{R_{v}\left(1+s\left(T_{i V}+\frac{1-H_{i}}{k_{i V} R_{v}}\right)+\frac{s^{2}}{\omega_{c I} k_{i V} R_{v}}\right)}{R_{t}\left(1+s T_{t}\right)\left(1+s T_{i V}\right)}
$$

Since $R_{v}$ is negative, the Nyquist criterion must be adopted to find the stability conditions: the Nyquist diagram shows that the ratio $\left|R_{v}\right| / R_{t}$ must be less than one (Fig. 5). The virtual resistance $R_{v}$ must be chosen so as to get a gain margin GM high enough: for instance higher than two.

A second loop is $F_{5}(s)$ in Fig. 4, where the feedback $H_{4}(s)$ is quite negligible $\left(\sin \delta^{0} \approx 0\right)$; thus this closed loop can be considered an open loop, which is stable if all its composing transfer functions are stable.

$T_{2 a}$ and $T_{2 b}$ do not depend on the load operating condition, but only on the parameters of the line $\left(R_{t}, L_{t}\right)$, of the controllers

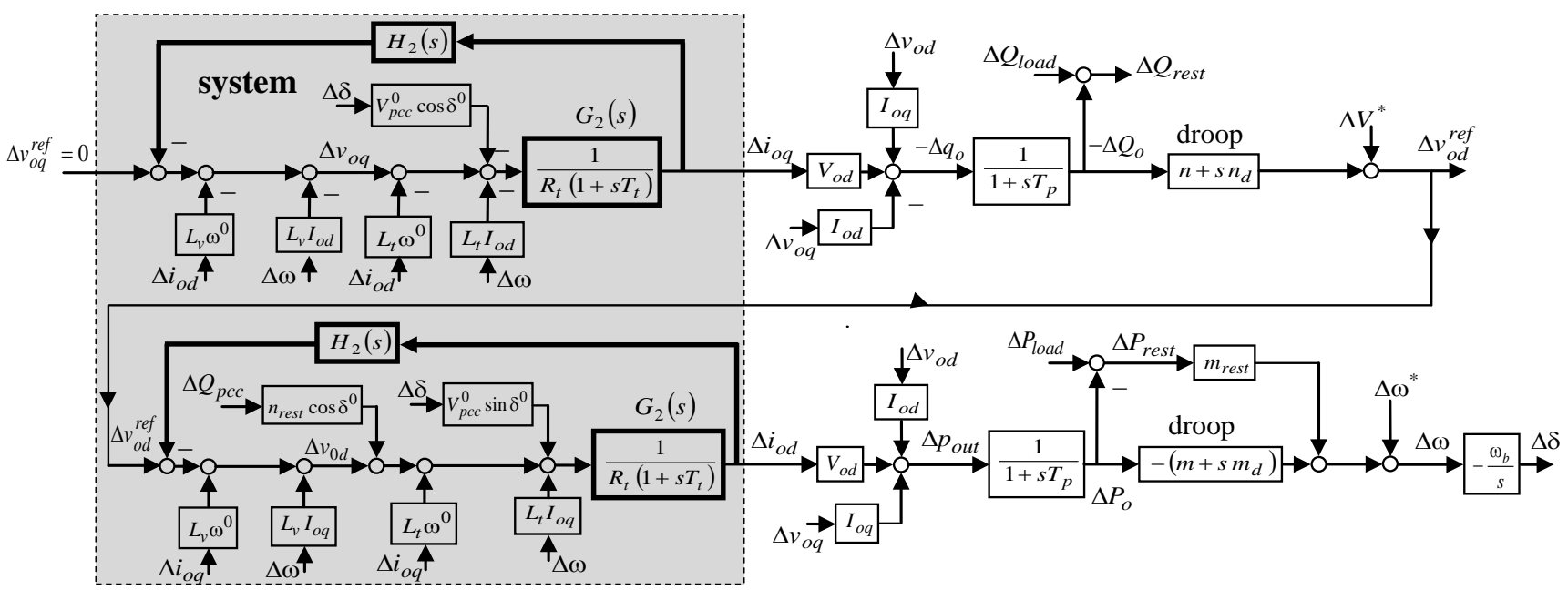

Fig. 3. Complete block diagram of the linearized system, assuming current loops with a bandwidth equal to $\omega_{c I}$. 


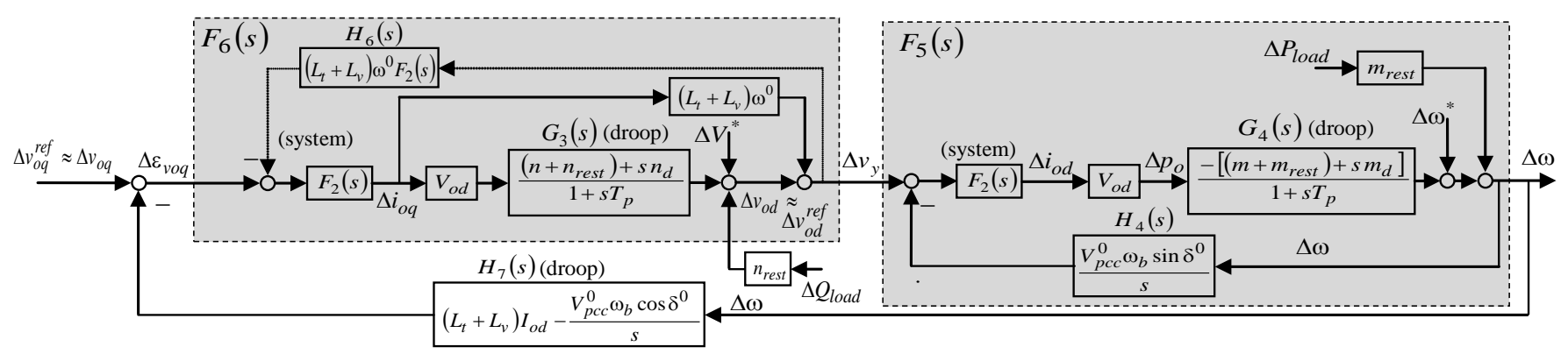

Fig. 4. Modification of the block diagram of Fig. 3, necessary to find the closed loop transfer functions $F_{5}(s)$ and $F_{6}(s)$.

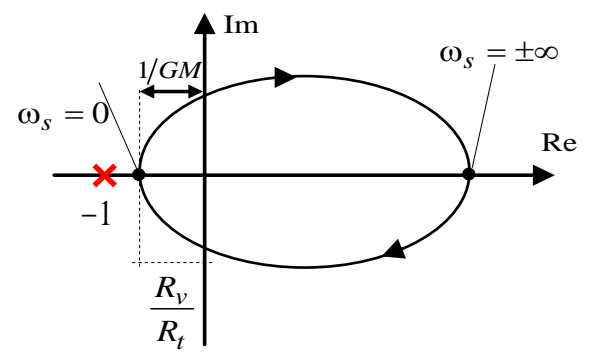

Fig.5. Nyquist diagram of $L_{2}(s)$ open loop transfer function: $R_{v} / R_{t}=-0.75$. GM is the gain margin.

A third loop is the subsystem $F_{6}(s)$ in Fig. 4: its feedback is depicted with a dotted line. By assuming:

$$
\begin{array}{r}
G_{3 b}(s)=V_{\text {od }} \frac{\left(n+n_{\text {rest }}\right)+s n_{d}}{1+s T_{p}}+\left(L_{t}+L_{v}\right) \omega^{0}= \\
=\mu_{G 3 b} \frac{1+s \tau_{G 3 b}\left(\tau_{d n}\right)}{1+s T_{p}} \\
\left.\mu_{G 3 b}=V_{\text {od }}\left(n+n_{\text {rest }}\right)+\left(L_{t}+L_{v}\right) \omega^{0}\right] \approx\left(L_{t}+L_{V}\right) \omega^{0} \\
\tau_{G 3 b}\left(\tau_{d n}\right)=\frac{V_{\text {od }} n \tau_{d n}+\left(L_{t}+L_{v}\right) \omega^{0} T_{p}}{V_{\text {od }}\left(n+n_{\text {rest }}\right)+\left(L_{t}+L_{v}\right) \omega^{0}}
\end{array}
$$

the subsystem changes as in Fig. 6. The open loop transfer function is:

$$
\begin{aligned}
& L_{6}(s)=F_{2}(s) G_{3 b}(s) H_{6}(s) \approx \\
& \quad \approx \frac{\mu_{G 3 b}\left(L_{t}+L_{v}\right) \omega^{0}}{\left(R_{t}+R_{v}\right)^{2}} \frac{\left(1+s T_{i V}\right)^{2}\left[1+s \tau_{G 3 b}\left(\tau_{d n}\right)\right]}{\left(1+s T_{2 a}\right)^{2}\left(1+s T_{2 b}\right)^{2}\left(1+s T_{p}\right)}
\end{aligned}
$$

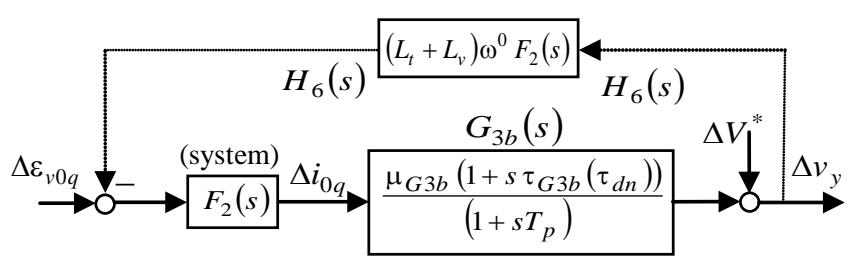

Fig. 6. Inner loop $\left(F_{6}\right)$ of the control system of Fig. 4.
This loop refers to the interaction between quantities on the $d$ and $q$ axes and does not exist in the decoupled SISO system used to design the controllers. The open loop transfer function $L_{6}(s)$ shows the main parameters which influence stability.

$>$ An increase of $L_{v}$ or a decrease of $R_{v}$ improves the decoupling between $P_{o}$ and $Q_{o}$ (Sect. III-B), but augments the gain of $L_{6}(s)$. This may lead the system to instability (Fig. 7a), because the phase margin PM is quite low.

$>$ Being $k_{p V}, k_{i V}$ constant, an increase of the current loop cutoff frequency $\omega_{c I}$ moves poles at $T_{2 b}{ }^{-1}$ to higher frequencies (see (27) ), thus improving stability (Fig. 7b) because the phase margin PM increases;

$>$ Changing $\tau_{d n}$ from positive (Fig. 7c) to negative (Fig. 7d) values moves the zero $\tau_{G 3 b}{ }^{-1}$ (32) to higher frequencies, close to the poles $T_{2 a}{ }^{-1}$; this improves stability, due to a rise of the phase margin PM;

$>$ An increase of $T_{i V}$, i.e. of the bandwidth of the voltage loop, moves the zeroes $T_{i V}{ }^{-1}$ leftwards (Fig. 7e), raising the phase margin PM and improving stability. This can be achieved by increasing $k_{p V}$ or decreasing $k_{i v}$. Nevertheless this adjustment implies that the pole $T_{2 a}{ }^{-1}$ moves leftwards (see (26)), lightly reducing the positive effect of the decrease of $T_{i V}{ }^{-1}$.

In order to analyze the external loop in Fig. 4, the transfer functions $F_{5}(s)$ between $\Delta v_{y}$ and $\Delta \omega$ as well as $F_{6}(s)$ between $\Delta \varepsilon_{v 0 q}$ and $\Delta v_{y}$ must be calculated. The open loop transfer function is:

$$
L_{7}(s)=F_{5}(s) F_{6}(s) H_{7}(s) \quad .
$$

An approximation formula of $L_{7}(s)$, valid for $\omega_{s}>T_{p}{ }^{-1}$, is:

$$
\begin{aligned}
L_{7}(s) \approx & \frac{V_{p c c}^{0} V_{0 d}\left(m+m_{\text {rest }}\right) \omega_{b}}{L_{t}+L_{v}} \cdot \frac{\cos \left(\delta^{0}\right)}{\omega^{0}} . \\
& \cdot \frac{1+s \tau d m}{s\left(1+s T_{p}\right)\left[\left(s / \omega_{0 F 6}\right)^{2}+2\left(\xi_{0 F 6} / \omega_{0 F 6}\right) s+1\right]}
\end{aligned}
$$

where:

$$
\xi_{0 F 6} \approx \frac{\left(R_{t}+R_{v}\right)}{\left(L_{t}+L_{v}\right) \omega^{0}} ; \omega_{0 F 6} \approx \frac{\left(L_{t}+L_{v}\right) \omega^{0}}{T_{p}\left(R_{t}+R_{v}\right)}
$$

The approximated open loop transfer function $L_{7}(s)$ and its bode diagram in Fig. 8 show that:

$>$ an increase of the gain of $L_{7}$ may lead to instability because the phase margin PM becomes low; such a gain depends on 
the squared voltage $V_{0 \mathrm{~d}} \approx V_{\text {pcc }}$, on the droop coefficients of both the line under study and the rest of the microgrid sources, $m$ and $m_{\text {rest }}$, and is inversely proportional to the inductance $L_{t}+L_{v}$

$>$ the damping $\xi_{0 F 6}$ should be large $(>1 / \sqrt{2})$ to avoid a resonant peak, that may intersect the $0 \mathrm{~dB}$ axis leading to instability;
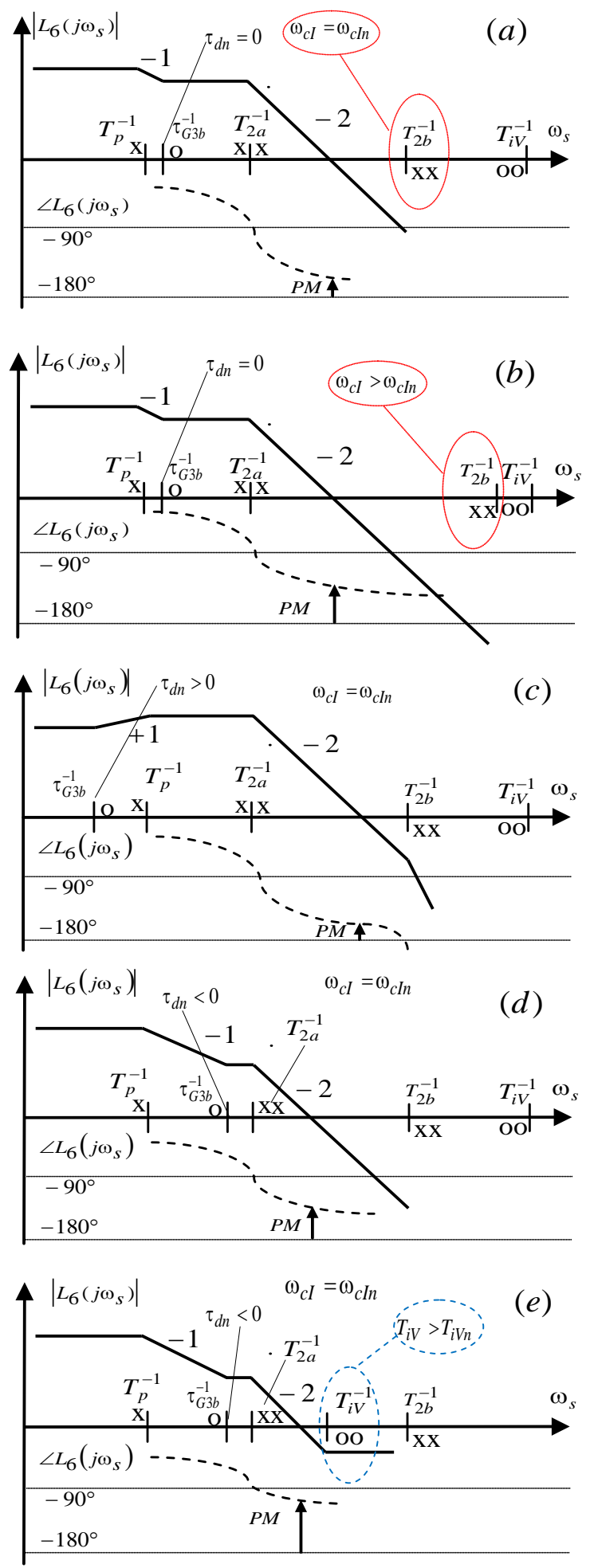

Fig. 7. Bode diagrams of the open loop transfer function $L_{6}(s)$. Description in the text. x: poles; o: zeroes. PM: phase margin.
$>$ in order to ensure stability, $\tau_{d m}$ should be chosen more than zero (intersection with the $0 \mathrm{~dB}$ axis with slope -1 ) and less than $T_{p}\left(\tau_{d m}{ }^{-1}>T_{p}{ }^{-1}\right)$ : in fact, this choice (unlike $\tau_{d m}{ }^{-1}<T_{p}{ }^{-1}$ ) would reduce the risk of a double intersection with the $0 \mathrm{~dB}$ axis due to the resonant poles $\omega_{0 F 6}$.

\section{B. Design guidelines}

The design of the voltage control parameters of an inverter to be connected to a microgrid in a droop-controlled mode starts from the basic transfer function of the voltage plant, neglecting mutual couplings between $d$ and $q$ axes. This is not enough to ensure stability, particularly when some virtual impedance is added. In a second step of the design, virtual impedance and derivative droop parameters must be taken into account and appropriate adjustments on the control parameters must be carried out. Some guidelines come from the previous analysis.

$\rightarrow$ Real time measurement of the line resistance $R_{t}$ and inductance $L_{t}$;

$>$ The values of the linear droop parameters $m$ and $n$ are chosen according to the well-known rules to get a correct power sharing with the other droop-controlled sources;

$>$ The virtual resistance $R_{v}$ and inductance $L_{v}$ should be chosen to get a sufficient decoupling of real and reactive powers:

o The virtual resistance $R_{v}$ should approach $-R_{t}$, keeping a good gain margin (Fig. 5): $\left|R_{v}\right|<0.75 R_{t}$.

o The virtual inductance $L_{v}$ must be chosen in connection to $R_{v}$, because the gain of $L_{6}(s)$ cannot increase too much to avoid a low phase margin (Fig. 7a);

o Additionally, a high $L_{v}$ would imply a higher voltage drop and a higher rated voltage of the converter: this is why it is preferable to use both the degrees of freedom $R_{v}$ and $L_{v}$ to ensure stability.

$>$ the integral time $T_{i V}$ of the voltage controller has to be corrected, so as to move the pole $T_{i V}{ }^{-1}$ between $T_{2 a}{ }^{-1}$ and $T_{2 b}{ }^{-1}$ (Fig. 7e); this implies a change in the bandwidth of the voltage loop;

$>$ the negative derivative droop coefficient $n_{d}$ applied to the reactive power affects time constant $\tau_{G 3 b}$ (32) through the parameter $\tau_{d n}=n_{d} /\left(n+n_{\text {rest }}\right): \tau_{G 3 b}$ is to be chosen so that $\tau_{G 3 b}{ }^{-1}>$ $T_{p}^{-1}$ and $\tau_{G 3 b} \approx T_{2 a}$ (Fig. $7 \mathrm{~d}$ );

$>$ the derivative droop coefficient $m_{d}$ applied to the active power is chosen so that $\tau_{d m}{ }^{-1}>T_{p}{ }^{-1}$ (Fig. 8).

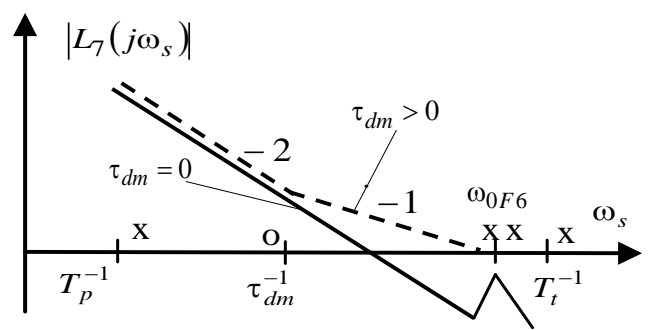

Fig. 8. Bode diagram of the open loop transfer function $L_{7}(s)$ when $\tau_{d m}=0$ (continuous line) and $\tau_{d m} \neq 0$ (dashed line). The case with $\tau_{d m}>0$ results in a more stable system. The saw-tooth in the continuous line is a resonant peak.

In case of large variations of the line impedance, these criteria 
can be adopted to implement an adaptive virtual impedance integrated with the real-time estimation of the line impedance seen from the capacitor terminals.

\section{NUMERICAL AND EXPERIMENTAL VALIDATION.}

A comparison between the proposed reduced model and experimental tests is now carried out. From the analysis of $L_{6}(s), L_{7}(s)$, the dominant poles and their damping factor $\xi_{d}$ of the related closed loop functions can be derived (Table II). These results are compared with the waveforms from the experimental tests.

\section{A. Setup parameters.}

As the experiments aim at validating the reduced-order model and design guidelines, and the real-time estimation of line impedance is beyond the scope of this paper, the system has been tested with different values of key design parameters, line impedance and constant virtual resistance and inductance. For these reasons, tests are carried out on a single inverter unit connected to the grid of the Lab: in other words, the droop coefficients of the other inverters are negligible $m_{\text {rest }}<<m$ and $n_{\text {rest }}<<n$ (Fig. 9). The grid stiffness is however adjusted by using different values of the line impedance to test a variety of conditions. The test perturbation used to assess stability is a sudden change in the real or reactive load powers $\Delta P_{\text {load }}$ $\Delta Q_{\text {load }}$ and this is practically achieved by varying the no-load voltage $V^{*}$ and angular frequency $\omega^{*}$ of the droop control (see inputs $\Delta V^{*}$ and $\Delta \omega^{*}$ in Fig. 4).

The validation of the model is carried out by analyzing the output power $P_{o}, Q_{o}$ response to an input step change in $\omega^{*}$ or $V^{*}$. In this case, it has been chosen to apply small positive steps starting from a no-load condition. The low value of the step amplitude avoids the saturation of the reference current (due to a limitation in the inverter's rating) and allows the validity conditions of the small signal analysis to be met.

The experimental system is characterized by:

$T_{t}^{-1}=625 \mathrm{rad} / \mathrm{s}, R_{v}=-0.75 R_{t} ; L_{v}=L_{t} ; m=0.01$ p.u.; $n=0.017$ p.u.; $\mu_{L 6}=15.9$ p.u. $=24.0 \mathrm{~dB}, \mu_{L 7}=65.2$ p.u. $=36.3 \mathrm{~dB}$ (gains of $L_{6}, L_{7}$ ).

\section{B. Discussion of results.}

The effect of a step in $\Delta V^{*}$ on the real and reactive powers is reported in Figs. 10 to 17, starting from $V^{*}=1.0025$ p.u., applying $\Delta V^{*}=0.01$ p.u. and using different control parameters as detailed below. Moreover, with a step in $\Delta \omega^{*}$, the system shows a low damping and wide oscillations, in case of no virtual impedance and no droop derivative parameters.

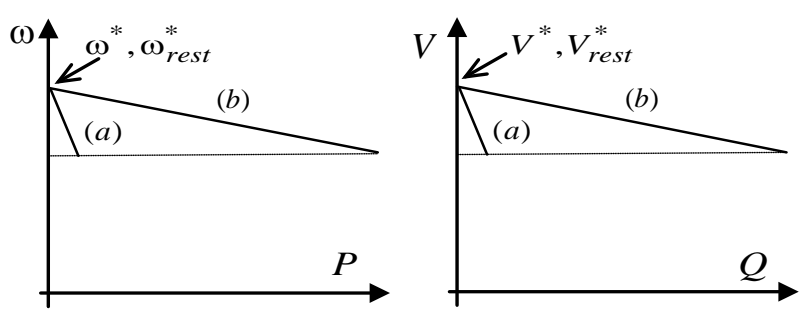

Fig. 9. Droop characteristics for the inverter under study (a) (described by (4), (5) ) and for the rest of the grid (b) (described by (9) ).
Case 1: with $\tau_{d m}=\tau_{d n}=0, \omega_{c I}=\omega_{c I n}, T_{i V}=T_{i V n}$, the results obtained by the reduced model show a very low damping ratio $\xi_{d}$ (3.3\%), which corresponds to a very low phase margin of $L_{6}(s)$ (as predicted in Fig. 7a).

The experimental test is reported in Fig. 10. The main parameters are: $V_{p c c}=1.0$ p.u.; $V^{*}=1.0025$ p.u. $\approx V_{p c c} ; \omega^{*} \approx 1.0008$ p.u.; $I_{0 d} \approx 0.08$ p.u.; $I_{0 q} \approx-0.04$ p.u.. $R_{v}=-0.75 R_{t}, L_{v}=L_{t}$. Before applying the $\Delta V^{*}$ step, an oscillation with a frequency close to the imaginary part of poles $p_{1,2}$ occurs (see Table II). After the step, another oscillation occurs, with a higher frequency but less than that exhibited by poles $p_{4,5}$. This situation corresponds to the case reported in Figs. 7a - 8. The real and reactive power $P_{o}, Q_{o}$ are well decoupled, as foreseen by the introduction of the virtual impedance (Sect. III). Anyway, power transients are characterized by significant oscillatory terms.

Case 2: like case 1, but with an increase in the cut off frequency $\omega_{c I}$ of the current loop $\left(\omega_{c I}=1.5 \omega_{c I I}\right)$; the reduced model shows a small improvement toward stability because poles $p_{1,2}$ move leftwards (as indicated in Fig. 7b). The experimental step-response is illustrated in Fig. 11 and shows a reduction in the oscillations.

Case 3: like case 1 , but $\tau_{d n}=2 T_{p}$; the reduced system shows instability: poles $p_{1,2}$ are in the right half plane as predicted in Fig. 7c. The experimental test shows an unstable behavior.

Case 4: like case 1 , but $\tau_{d n}=-2.2 T_{p} ; \tau_{d n}$ is chosen so that the zero $\tau_{G 3 b}{ }^{-1}$ of $L_{6}(s)$ is close to the pole $T_{2 a}{ }^{-1}$. In this case, the reduced model is stable, but two poles $p_{4,5}$ are still located close to the imaginary axis (i.e. with a low damping coefficient). They are introduced by $L_{7}(s)$ (Fig. 8). The experimental test (Fig. 12) shows the reduction of high frequency oscillations but not a reduction of low frequency ones.

Case 5: like case 1 , but $\tau_{d n}=-2 . T_{p}$ and $T_{i V}=2.5 T_{i V n}$ (increase of the bandwidth of the voltage loop, by increasing $T_{i V}$ ); in the open loop transfer function $L_{6}(\mathrm{~s})$, the two zeroes $T_{i V}{ }^{-1}$ move leftwards (Fig. 7d), improving the phase margin. The poles $p_{1,2}$ of the closed loop function move leftwards (Table II); but not poles $p_{4,5}$ (again introduced by $L_{7}(s)$ ).

The corresponding test is shown in Fig. 13 and reveals oscillations at low frequency, close to the imaginary part of poles $p_{4,5}$; with respect to Fig. 10, however, high frequency oscillations have disappeared.

Case 6: the same as case 5 plus $\tau_{d m}=0.4 T_{p}$; all the poles in table II are quite stable as the transfer function $L_{7}(s)$ states out (see Figs. 7e - 8). Figs. 14(a) and 14(b) show the experimental response to a step in $\Delta V^{*}$ and in $\Delta \omega^{*}$ respectively: the system behaves quite fairly as predicted in Table II by the reduced model, and the oscillations are more bounded. This corresponds to the best condition for the parameters design, coherently to the proposed simplified model. 
A further comparison between complete and reduced models is done considering the eigenvalues of the simplified model derived from transfer function analysis and those obtained from the complete set of equations (1)-(10) when $\tau_{d n}$ is changed in the range $\left[-2.2 T_{p}, 2 T_{p}\right]$. This includes by the way cases 4, 3 and 1 in Table II. Dots in Fig. 15 are the dominant eigenvalues $p_{1,2}$ and $p_{3}$ of the reduced model, whereas stars represents the corresponding eigenvalues of the full model (1)(10). The comparison is shown in Fig. 15 and reveals that the proposed model and design technique can predict the dynamic properties of the system.

Another test has been carried out with tripled line impedance $R_{t}, L_{t}$. Obviously, the voltage control parameters must be changed in order to get the stability and this should be carried out with an adaptive control. Nevertheless the criteria described in Sect. V.B turn out to be still valid, as shown in Figs. 16-17.

\section{TABLE II}

DOMINANT POLES $p_{1,2}, p_{3}, p_{4,5}$ AND DAMPING COEFFICIENT $\xi_{d}$ FROM THE REDUCED MODEL (FIG. 3), IN A QUASI-NO-LOAD CONDITION AND WITH DIFFERENT CONTROL AND LINE PARAMETERS. IN BOLD THE PARAMETERS AND DOMINANT POLES, WHICH HAVE CHANGED FROM THE PRECEDING CASE.

\begin{tabular}{|c|c|c|c|c|}
\hline case & Figure & $\begin{array}{c}\text { Poles and zeroes of } L_{6} \text {, } \\
L_{7}[\mathrm{rad} / \mathrm{s}]\end{array}$ & $\begin{array}{l}p_{1,2}-p_{3-} p_{4,5} \\
{[\mathrm{rad} / \mathrm{s}]}\end{array}$ & $\begin{array}{c}\xi_{d} \\
{[\%]}\end{array}$ \\
\hline 1 & $\begin{array}{l}7 \mathrm{7a} \\
10\end{array}$ & $\begin{array}{l}T_{p}^{-1}=10 \\
\tau_{G 3 b}{ }^{-1}=13\left(\rightarrow \tau_{d n}=0\right) \\
T_{2 a}{ }^{-1}=65 ; T_{2 b}^{-1}=242 ; \\
T_{i V}{ }^{-1}=500 \\
\tau_{d m}{ }^{-1}=\infty\end{array}$ & $\begin{array}{l}-6.0 \pm j 181^{(1)} \\
-13.3^{(1)} \\
-1.72 \pm j 256^{(2)}\end{array}$ & $\begin{array}{l}3.3 \\
100 \\
0.66\end{array}$ \\
\hline 2 & $7 \mathrm{~b}$ & $T_{2 b}^{-1}=359$ & $\begin{array}{l}-\mathbf{- 3 0} \pm \mathbf{j 1 9 3}^{(1)} \\
-13.3^{(1)} \\
-2.1 \pm j 258^{(2)}\end{array}$ & 15 \\
\hline 3 & $7 \mathrm{c}$ & $\begin{array}{l}\tau_{G 3 b}^{-\mathbf{1}}=\mathbf{8 . 0} \\
\left(\rightarrow \tau_{d n}=2 . T_{p}\right) ;\end{array}$ & $\begin{array}{l}+\mathbf{+ 6 . 5} \pm \mathbf{j} 22 \mathbf{1}^{(1)} \\
-8.0^{(1)} \\
-1.7 \pm \mathrm{j} 256^{(2)}\end{array}$ & -2.9 \\
\hline 4 & $7 \mathrm{~d}$ & $\begin{array}{l}\tau_{G 3 b}{ }^{-1}=\mathbf{5 6} \\
\left(\rightarrow \tau_{d n}=-2.2 T_{p}\right)\end{array}$ & $\begin{array}{l}\mathbf{- 1 8} \pm \mathbf{j} 99^{(1)} \\
-\mathbf{5 7} 7^{(1)} \\
-1.7 \pm j 256^{(2)}\end{array}$ & $\begin{array}{l}18 \\
100\end{array}$ \\
\hline 5 & $7 \mathrm{e}$ & $\begin{array}{l}T_{2 a}^{-1}=55 ; T_{2 b^{-1}}=217 \\
\boldsymbol{T}_{i V^{-1}}=\mathbf{2 0 0}\end{array}$ & $\begin{array}{l}-\mathbf{- 4 4} \pm \mathbf{j} 103^{(1)} \\
-43.3^{(1)} \\
-2.5 \pm j 26^{(2)}\end{array}$ & 39 \\
\hline 6 & $\begin{array}{l}\text { 7e, } \\
8\end{array}$ & 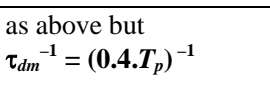 & $\begin{array}{l}-44 \pm \mathrm{j} 103^{(1)} \\
-43.3^{(1)} \\
-\mathbf{2 0} \pm \mathbf{j} 19^{(2)}\end{array}$ & 72 \\
\hline
\end{tabular}

(1), (2) refer to the closed loop transfer functions (t.f.) $F 6, F 7$ obtained by the open loop t.f.s $L 6, L 7$ respectively.

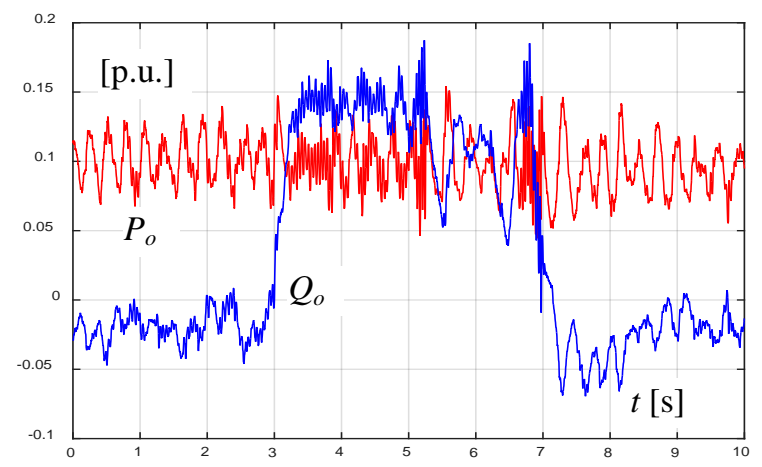

Fig. 10. Case 1): real $P_{o}$ and reactive $Q_{o}$ power waveforms as a response to a step in the $\mathrm{v}_{\text {odref }}-Q_{o}$ droop curve. The step is obtained by changing $V^{*}$ by $1 \mathrm{E}-2$ p.u. .

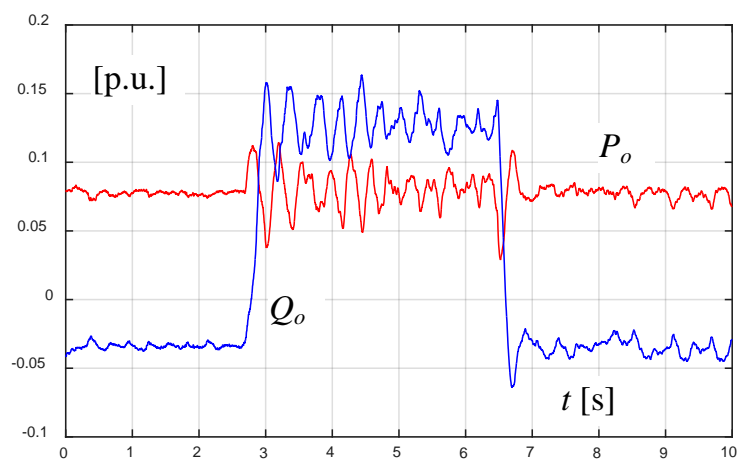

Fig. 11. Case 2): step perturbation as in Fig. 10.

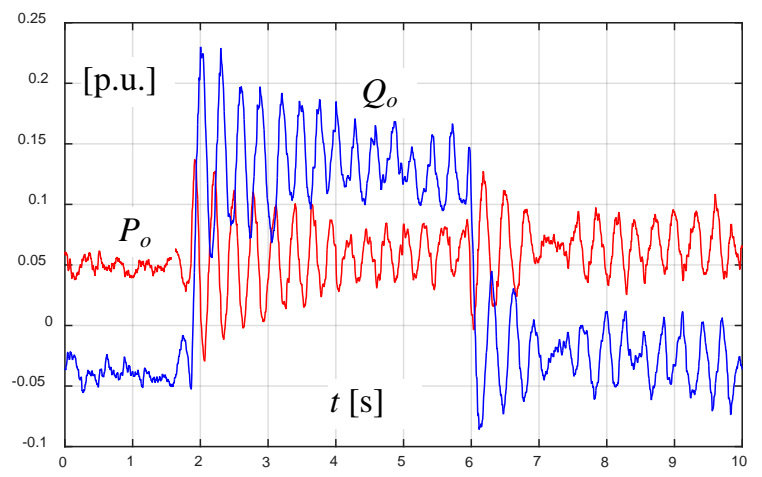

Fig. 12. Case 4): step perturbation as in Fig. 10.

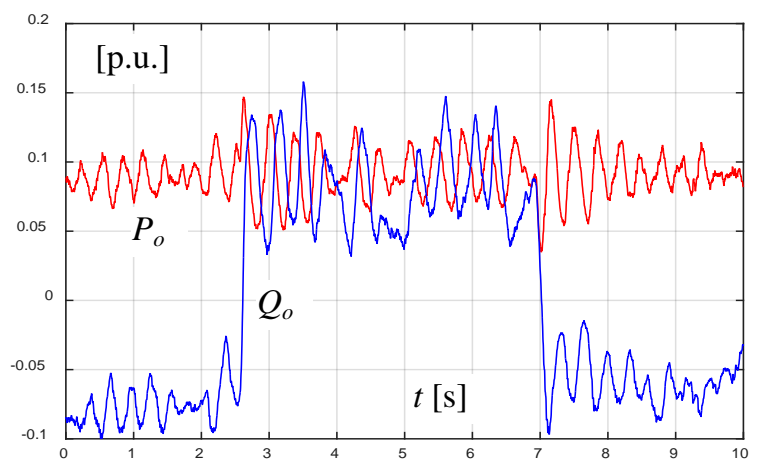

Fig. 13. Case 5): step perturbation as in Fig. 10.

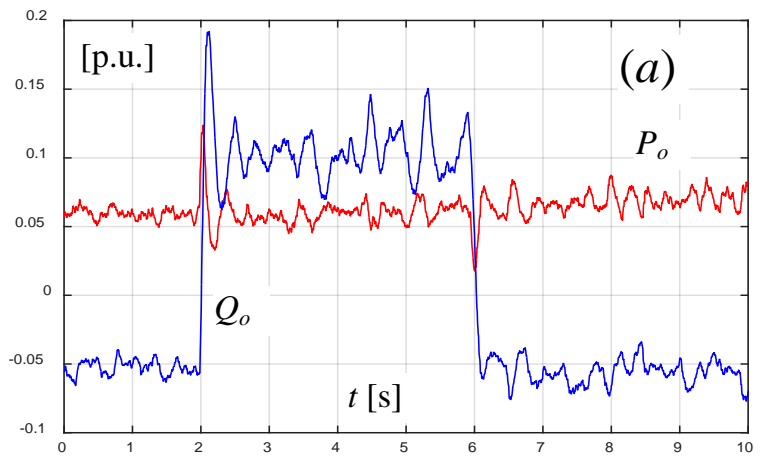




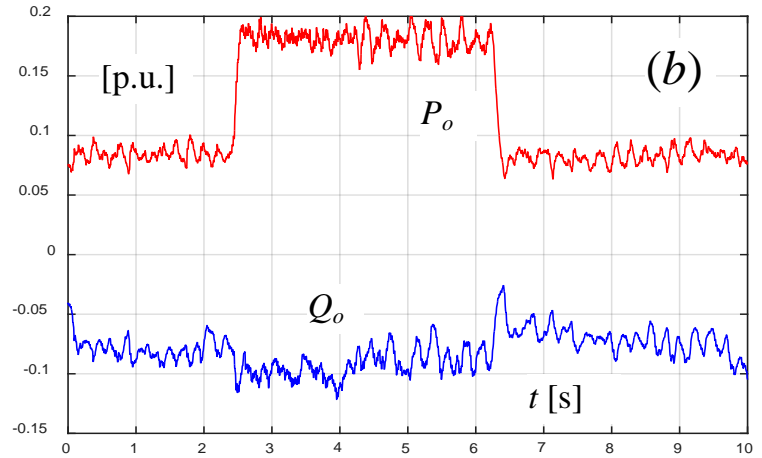

Fig. 14. Case 6). Real $P_{o}$ and reactive $Q_{o}$ power waveforms as a response to a step in the $\mathrm{v}_{\text {odref }}-Q_{o}$ droop (a) and in the $\omega-P$ one (b) , respectively. As for the latter, the step is obtained by changing $\omega^{*}$ by $1 \mathrm{E}-3$ p.u..

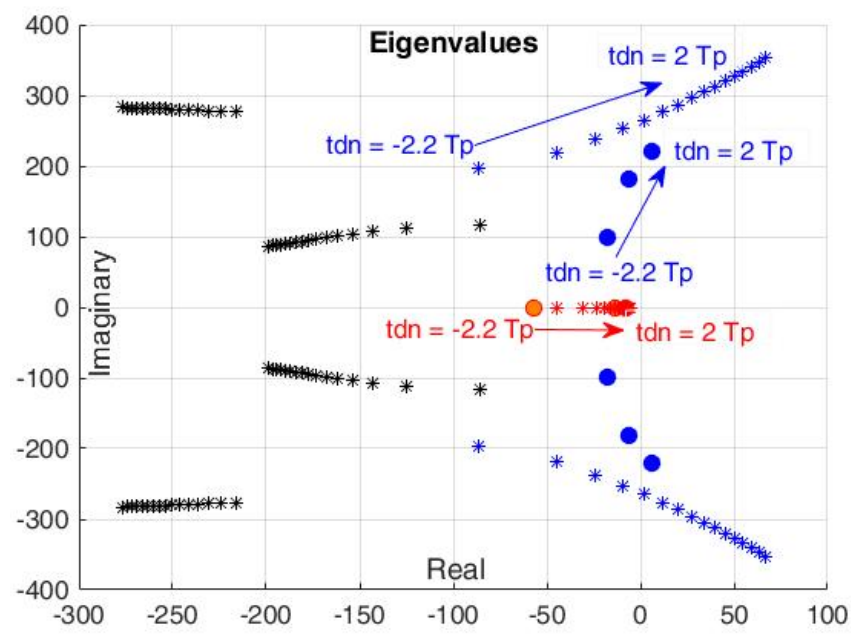

Fig. 15. Eigenvalues comparison of the reduced model with the complete one, when the parameter $\tau_{d n}$ is changed in the range $\left[-2.2 T_{p} ; 2 T_{p}\right]$ : dots = reduced model eigenvalues $\left(p_{1,2}-p_{3}\right)$ stars $=$ full model $(1)-(10)$. The proposed reduced model is able to effectively keep track of the dominant dynamics in the system. The reduced-model eigenvalues $p_{4,5}$ as well as the corresponding dynamics of the complete model are not influenced by the parameter $\tau_{d n}$; thus they are not included in the graph.

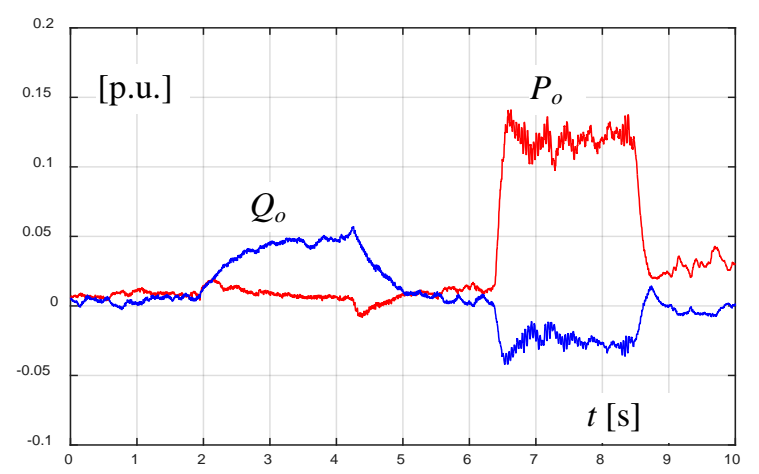

Fig. 16. As Fig. 10, but with tripled line impedance. $\tau_{d m}=0.4 T_{p} . \tau_{d n}=0$. The comparison with the case in the next figure shows the importance to choose a negative derivative reactive droop parameter $\tau_{d n}$. Here are reported the transients associated respectively to a step changes of $V^{*}$ by $1 \mathrm{E}-2$ p.u. and $\omega^{*}$ by $1 \mathrm{E}-3$ p.u.

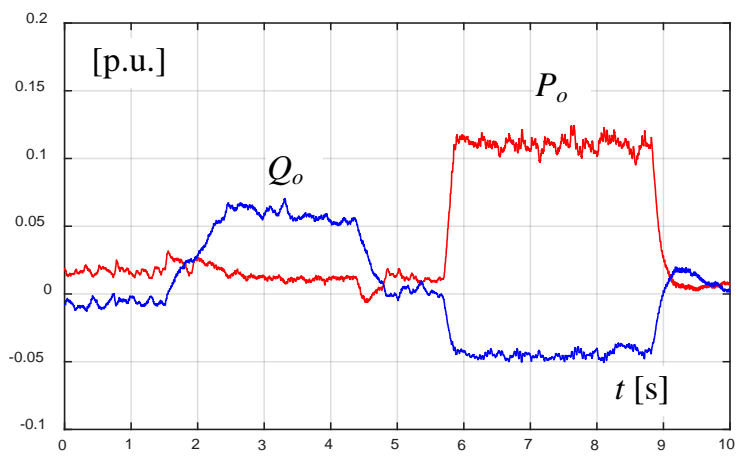

Fig. 17. As Fig. 16 (tripled line impedance) but with $\tau_{d m}=0.4 T_{p} . \tau_{d n}=-2 T_{p}$. It corresponds to case 6). As before, the two transients correspond to step change of $V^{*}$ by $1 \mathrm{E}-2$ p.u. and $\omega^{*}$ by $1 \mathrm{E}-3$ p.u.

\section{CONCLUSIONS}

The paper has derived a comprehensive transfer-function model for a droop-controlled VSI with filter and interface transformer operating in parallel to a microgrid comprising other units and loads. This structure can be regarded as the elementary unit in a microgrid. The equations of the full statespace model have been simplified and manipulated in order to represent the model as a series of single-input, single-output sub-systems with no interlaced connections. In spite of the simplifications, the derived model has still enough "structure" to represent interactions between control loops, droop and virtual impedance. Furthermore, the model lends itself to an explicit analytical stability analysis. The impact of the main control, droop and virtual impedance parameters on stability has been investigated and criteria for a coordinated choice of these parameters have been derived. In particular, it is recommended to adopt both the resistive and inductive components in the virtual impedance, in such a way as to preserve stability with minimum voltage drop and VSI ratings. Furthermore, the integral time $T_{i V}$ of the voltage controller designed according to the traditional decoupled, nested-loop plant-scheme has to be corrected in relation to the virtual impedance values, and delimitations are found for the optimal derivative droop time-constants $\tau_{d m}$ and $\tau_{d n}$ as a function of virtual impedance, power filter time-constant and cut-off frequency of current loops.

Based on the comparison of the predicted dominant poles to the experimental behavior of a $2.4 \mathrm{kVA}$ setup, the model proves to be fairly accurate in evaluating stability margins in different scenarios and can be proposed as an effective design and analysis tool.

\section{REFERENCES}

[1] M. Savaghebi; A. Jalilian; J. C. Vasquez; J. M. Guerrero "Autonomous Voltage Unbalance Compensation in an Islanded Droop-Controlled Microgrid”, IEEE Trans. on Ind. El 2013, Vol 60, Issue 4, pp. 13901402

[2] E. Planas, A. Gil-de-Muro, J. Andreu, I. Kortabarria, I.Martinez de Alegria, " Design and implementation of a droop control in a d-q frame for islanded microgrid”, IET Renew. Power Gener., 2013, vol. 7, Iss. 5, pp.458-474. 
[3] T.L. Vandoorn, B. Meersman, J.D.M. De Kooning, L. Vandevelde, "Transition from islanded to grid-connected mode of microgrids with voltage based droop control, IEEE Trans. on Power Systems, Vol. 28, No.3, Aug. 2013, pp. 2545-2553

[4] C. N. Rowe, T. J. Summers, R. E. Betz, D. J. Cornforth, T. G. Moore, "Arctan power-frequency droop for improved microgrid stability", IEEE Trans. on Power. Electronics, Vol. 28, No.8, Aug. 2013

[5] J.P. Guerrero, L. Garcia de Vicuna, J. Matas, M.Castilla, J. Miret, “A wireless controller to enhance dynamic performance of parallel inverters in distributed generation systems", IEEE Trans. on Power Electronics, Vol. 19, No.5, Sept. 2004, pp. 1205-1213

[6] Y. A. I. Mohamed, E. F. El-Saadany, "Adaptive decentralized droop controller to preserve power sharing stability of parallel inverters in distributed generation microgrids", IEEE Trans. on Power Electronics, Vol. 23, No.6, Nov. 2008

[7] X. Yu, A.M. Khambadkone, H. Wang, S.T.S. Terence, “ Control of paralle- connected power converters for low voltage microgrid - Part I: a hybrid control architecture", IEEE Trans. on Power Electronics, Vol. 25, No.12, Dec. 2010, pp. 1962-2970

[8] H. Mahmood, D. Michaelson, J Jiang, "Accurate Reactive Power Sharing in an Islanded Microgrid Using Adaptive Virtual Impedances", IEEE Trans. on Power El. 2015, Vol. 30, Issue 3 pp. 1605 - 1617.

[9] K. De Brabandere, B. Bolsen, J. Van der Keybus, A. Woyte, J. Driesen, R. Bemans, " A voltage and frequency droop control method for parallel inverters”, IEEE Trans. on Power Electronics, Vol. 22, No.4, July 2007

[10] T. L. Vandoorn, J. D. M. De Kooning, B. Meersman, J. M. Guerrero and L. Vandevelde, "Automatic Power-Sharing Modification of Droop Controllers in Low-Voltage Resistive Microgrids," IEEE Trans. on Power Delivery, vol. 27, no. 4, pp. 2318-2325, Oct. 2012.

[11] J.P. Guerrero, L. Garcia de Vicuna, J. Matas, M.Castilla, J.Miret, "Output impedance design of parallel-connected UPS inverters with wireless load sharing control”, IEEE Trans. on Ind. Electronics, Vol. 52, No.4, Aug. 2005, pp. 1126-1135

[12] J. He, Y. W. Li, "Analysis, design, and implementation of virtual impedance for power electronics interfaced distributed generation", IEEE Trans on Ind. Appl., Vol. 47, N. 6, Nov/Dec 2011, pp. 25252538.

[13] J. He, Y. Wei Li, J.P. Guerrero, F. Blaabjerg, J.C. Vasquez, “An islanding microgrid power sharing approach using enhanced virtual impedance control scheme”, IEEE Trans. on Power Electronics, Vol. 28, No.11, Nov. 2013

[14] W. Yao, M. Chen, J. Matas, J.M. Guerrero, Z. Quiang, “ Design and analysis of the droop control method for parallel inverters considering the impact of the complex impedance on the power sharing”, IEEE Trans. on Ind. Electronics, Vol. 58, No.2, Feb. 2011, pp. 576-587

[15] A. El Boubakri, L.A.C. Lopez, "Phase shift based equivalent virtual impedance loop for droop-controlled inverters", $16^{\text {th }}$ European Conf. on Power Electronics and Applications EPE 2014, Lappeenranta Finland 26-28 Aug. 2014.

[16] X. Tang, W. Deng, Z. Qi, "Investigation of the dynamic stability of microgrid”, IEEE Trans. on Power Systems, Vol. 29, No.2, Mar. 2014, pp. $698-706$.

[17] Md. Rasheduzzaman, J. A. Mueller, J. W. Kimball, “ Reduced-order, small-signal model of microgrid systems”, IEEE Trans. on Sustainable Energy, Vol. 6, No.4, Oct. 2015, pp. 1292 - 1304.

[18] K. Yu, S. Wang, J. Ni, T. Lv, "Analysis and optimization of droop controller for microgrid system based on small-signal dynamic model”, IEEE Trans. on Smart Grid, Vol. 7, No.2, Mar. 2016, pp. 695 - 704.

[19] X. Tang, X. Hu, N, Li, W. Deng, G. Zhang, "A novel frequency and voltage control method for islanded microgrid based on multienergy storages”, IEEE Trans. on Smart Grid, Vol. 7, No.1, Jan. 2016, pp. 410 -419 .

[20] M. Liserre, F. Blaabjerg, S. Hansen, “ Design and control of an LCLfilter-based three-phase active rectifier”, IEEE Trans. on Ind. Appl., Vol. 41, No.5, Sept/Oct. 2005, pp. $1281-1290$

[21] N. Pogaku, M. Prodanovic, T. Green, “ Modeling, analysis and testing of autonomous operation of an inverter-based microgrid", IEEE Trans. on Power Electronics, Vol. 22, No.2, Mar. 2007, pp. 613 - 625.

\section{BIOGRAPHIES}

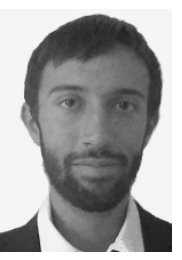

Alberto Bolzoni (St. M'17) received his MS degree in electrical engineering at Politecnico di Milano, Milano, Italy, in 2015.

Currently, he is a Ph. D. student at the Department of Energy at Politecnico di Milano, and his main field of interest is power electronics applied to isolated microgrids.

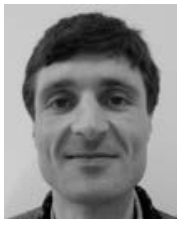

Giovanni Maria Foglia received his MS degree and the $\mathrm{PhD}$ in electrical engineering at Politecnico di Milano, Milano, Italy, in 1997 and 2000.

Currently, he is an Assistant Professor at the Department of Energy at Politecnico di Milano, and his main field of interest is the analysis and design of PM electrical machines.

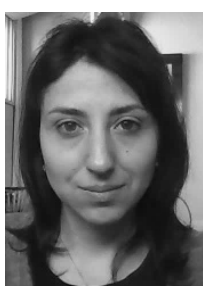

Luisa Frosio received the $\mathrm{PhD}$ in electrical engineering from the Politecnico di Milano, Milano, in 2012. Currently, she is working as Business Development Manager in EPS Electro Power Systems. She is in charge of the technical and economical design and project assessment for battery energy storage systems and hybrid power plants in on grid and off grid applications.

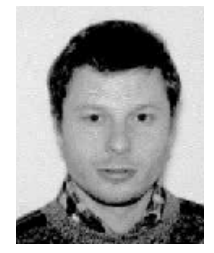

Matteo Felice Iacchetti (M'10) received the PhD in electrical engineering from the Politecnico di Milano, Milano, in 2008.

Currently, he is a Lecturer with the School of Electrical and Electronic Engineering at The University of Manchester, Manchester, UK. His main research interests are the design, modeling and control of electrical machines.

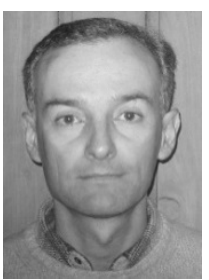

Roberto Perini (M'10) received his MS degree and the $\mathrm{PhD}$ in electrical engineering from the Politecnico di Milano, Milano, Italy.

Currently, he is an Associate Professor at the Department of Energy at Politecnico di Milano. His interests are in the design and modeling of electrical machines and microgrids. 